\title{
Blockade of RAGE suppresses periodontitis-associated bone loss in diabetic mice
}

\author{
Evanthia Lalla, ${ }^{1}$ Ira B. Lamster, ${ }^{1}$ Michael Feit,${ }^{1}$ Linda Huang, ${ }^{1}$ \\ Alexandra Spessot, ${ }^{1} \mathrm{Wu}$ Qu, ${ }^{2}$ Thomas Kislinger, ${ }^{2}$ Yan Lu, ${ }^{2}$ \\ David M. Stern, ${ }^{2}$ and Ann Marie Schmidt ${ }^{2}$ \\ ${ }^{1}$ Division of Periodontics, School of Dental and Oral Surgery, and \\ ${ }^{2}$ Departments of Physiology, Surgery, and Medicine, College of Physicians and Surgeons, Columbia University, \\ New York, New York 10032, USA
}

Address correspondence to: Evanthia Lalla, Division of Periodontics, Columbia University School of Dental and Oral Surgery, 630 W. 168th Street, PH-7E-110, New York, New York 10032, USA.

Phone: (212) 305-9283; Fax: (212) 305-9313; E-mail: el94@columbia.edu.

Received for publication November 18, 1999, and accepted in revised form February 3, 2000.

\begin{abstract}
Diabetes is associated with increased prevalence, severity, and progression of periodontal disease. To test the hypothesis that activation of RAGE (Receptor for Advanced Glycation End products) contributes to the pathogenesis of diabetes-associated periodontitis, we treated diabetic mice, infected with the human periodontal pathogen Porphyromonas gingivalis, with soluble RAGE (sRAGE). sRAGE is the extracellular domain of the receptor, which binds ligand and blocks interaction with, and activation of, cell-surface RAGE. Blockade of RAGE diminished alveolar bone loss in a dose-dependent manner. Moreover, we noted decreased generation of the proinflammatory cytokines TNF- $\alpha$ and IL- 6 in gingival tissue, as well as decreased levels of matrix metalloproteinases. Gingival AGEs were also reduced in mice treated with sRAGE, paralleling the observed suppression in alveolar bone loss. These findings link RAGE and exaggerated inflammatory responses to the pathogenesis of destructive periodontal disease in diabetes.
\end{abstract}

J. Clin. Invest. 105:1117-1124 (2000).

\section{Introduction}

Multiple studies have demonstrated conclusively a link between diabetes and periodontal disease in human subjects $(1,2)$. Consequently, periodontal disease has been described as the sixth complication of diabetes mellitus (3). It is likely that multiple factors underlie this association, from impaired host defenses upon engagement of invading bacterial pathogens to exaggerated inflammatory responses once infection is established. Potential pathogenic mechanisms for accelerated alveolar bone loss in diabetic subjects include impaired recruitment and function of neutrophils in response to infection with pathogenic bacteria $(4,5)$, diminished generation of collagen and exaggerated collagenolytic activity $(6,7)$, as well as genetic predisposition (8). Recent studies have suggested that the enhanced inflammatory response to periodontal pathogens is associated with increased severity of diabetic periodontal disease (9). Specifically, elevated levels of inflammatory mediators, including increased production of prostaglandin E2 (PGE2), IL- $1 \beta$, and TNF- $\alpha$ by monocytes in culture, as well as increased levels of PGE2 and IL- $1 \beta$ in gingival exudate, were observed in diabetic patients with gingivitis or periodontitis compared with age-matched non-diabetic controls (10).

Even sporadically elevated levels of blood glucose lead to the generation of largely irreversible Advanced Glyca- tion End products, or AGEs (11-13). AGEs accumulate in the tissues and plasma of humans and rodents in normal aging and do so to a greater degree in those with diabetes. Their earlier and enhanced accumulation in diabetes has been linked to the pathogenesis of vascular and inflammatory cell complications that typify this disorder. Our laboratory has identified a central cell-surface receptor for these AGEs, termed RAGE (receptor for AGE), a multiligand member of the immunoglobulin superfamily of cell-surface molecules $(14,15)$. RAGE is expressed on a range of cell types, such as endothelial cells, monocytes, smooth muscle cells, and fibroblasts, at low levels in adult healthy animals but at significantly higher levels in settings such as diabetes (16-18). Multiple studies have demonstrated that engagement of RAGE by AGEs on cells critically involved in inflammatory responses results in cellular perturbation (19). For example, in endothelial cells interaction of RAGE with AGEs results in hyperpermeability and enhanced expression of a range of adhesion molecules such as vascular cell adhesion molecule-1 (VCAM-1). AGE-RAGE interaction on monocytes induces chemotaxis and haptotaxis, as well as increased generation of cytokines such as TNF- $\alpha$, IL-1 $\beta$, and IL- 6 . Further, in fibroblasts engagement of RAGE results in diminished collagen synthesis. Indeed, evidence for perturbed cellular and connective tissue properties has been established in the setting of long-term diabetes (20). 
Consistent with an important role for AGE-RAGE interaction in diabetic complications, in vivo studies revealed that administration of soluble RAGE (sRAGE), the extracellular ligand-binding domain of RAGE, to diabetic rodents resulted in diminished vascular hyperpermeability and suppression of accelerated atherosclerosis $(21,22)$. In the latter case, treatment of diabetic apo E-null mice with sRAGE resulted in quenching of cellular activation, with decreased numbers of monocytes and smooth muscle in lipid/AGE-rich atheroma (22).

Our recent observations have suggested that RAGE is also a central cell-surface receptor for EN-RAGE (extracellular newly identified RAGE binding proteins) and other members of the S100/calgranulin family of proinflammatory cytokines (23). These largely intracellular proteins (within effector cells key in the inflammatory response such as polymorphonuclear leukocytes and monocytes) may gain access to the extracellular space in inflammatory milieu. Upon release, their ability to interact with cellular RAGE appears to be an important means by which to propagate and sustain inflammatory cellular perturbation and chronic tissue injury $(24,25)$. Consistent with these concepts, blockade of RAGE and/or EN-RAGEs suppressed inflammation and activation of key transcription factors such as NF- $\mathrm{\kappa B}$ in murine models of delayed-type hypersensitivity and colitis (23).

We thus hypothesized that the interaction of RAGE with AGEs and/or EN-RAGEs may contribute to inflammation and destruction of nonmineralized connective tissue and bone in diabetes-associated periodontal disease. To test this hypothesis, we previously established a model of periodontal inflammation and accelerated alveolar bone loss in diabetic mice infected by oral/anal administration with the human Gram-negative anaerobic periodontal pathogen Porphyromonas gingivalis (26). Here we demonstrate that blockade of RAGE in this murine model diminished accelerated alveolar bone loss. Importantly, decreased generation of inflammatory cytokines and tissue-destructive matrix metalloproteinases (MMPs) was observed in the presence of RAGE blockade. These findings were associated with decreased levels of gingival tissue AGEs in a manner paralleling the suppression of alveolar bone loss. These data implicate RAGE and exaggerated inflammatory responses in the pathogenesis of destructive periodontitis associated with diabetes.

\section{Methods}

Induction of diabetes and assessment of byperglycemia. C57BL/6 male mice were obtained from The Jackson Laboratories (Bar Harbor, Maine, USA) and housed in a standard non-pathogen-free environment. When the mice were 6-7 weeks old, diabetes was induced by 4 daily intraperitoneal injections of streptozotocin (55 mg/kg; Sigma Chemical Co., St. Louis, Missouri, USA) diluted into sterile citrate buffer $(0.05 \mathrm{M}, \mathrm{pH} 4.5)$. Control mice were injected with citrate buffer alone. Seven days after the first injection of either streptozotocin or citrate buffer, non-fasted whole blood $(0.05 \mathrm{~mL})$ was removed from the tail vein. Plasma was separated from the red blood cells and assessed for levels of glucose using the glucose oxidase method (Sigma). Analysis of plasma glucose was repeated on an alternate day. Diabetes, mediated by relative insulin deficiency, was defined as serum glucose $\geq 300 \mathrm{mg} / \mathrm{dL}$ on 2 separate occasions. Animals treated with streptozotocin that did not manifest hyperglycemia (approximately 5\%) were treated with 2 further daily injections of streptozotocin. Assessment of serum glucose was repeated 3 days later and mice who did not become diabetic were sacrificed ( $<1 \%$ of latter group). At sacrifice, red blood cells were removed, washed, and lysed for detection of levels of glycosylated hemoglobin (Pierce Chemical Co., Rockville, Illinois, USA).

Inoculation with Porphyromonas gingivalis. One month after treatment with streptozotocin or citrate buffer, mice were infected with $P$. gingivalis, strain 381, generously provided by J. Zambon (State University of New York, Buffalo, New York, USA) $(27,28)$. Approximately $0.2 \mathrm{~mL}$ of $1.5 \times 10^{12}$ cells $/ \mathrm{mL}$ in PBS were administered every other day for a total of 4 days, in part by gavage, and by local application using a cotton swab along the gingival margin throughout the mouth and the colorectal area (26-28). Mice were then allowed free access to standard mouse chow (Purina 5001, Purina Mills Inc., St. Louis, Missouri, USA) and water.

Preparation and administration of murine sRAGE. Murine sRAGE was prepared in a baculovirus expression system using Sf9 cells, purified to homogeneity, devoid of endotoxin, and sterile-filtered $(0.2 \mu \mathrm{m})$ according to procedures published previously (22). Mice received daily intraperitoneal injections of sRAGE (at dosages ranging from 3.5 to $100 \mu \mathrm{g}$ per day), commencing the day after administration of $P$. gingivalis was completed and continuing for a total of 2 months. This time point was previously shown to be the earliest one at which bone loss became significantly greater in diabetic mice as compared with non-diabetic controls (26). Murine serum albumin (MSA; Sigma) was employed as control treatment.

Quantification of alveolar bone loss. Evaluation of the extent of periodontal destruction by measurement of alveolar bone loss was determined as described previously (26). Briefly, at sacrifice mandibles were removed, hemisected, exposed to $\mathrm{KOH}(2 \%)$, and then mechanically defleshed. The jaws (exposure of the lingual surface of each half mandible) were photographed using a dissecting microscope. For each mouse, alveolar bone loss was defined as the total area between the cemento-enamel junction (CEJ) and the alveolar bone crest (BC) for a total of 6 posterior teeth. The slides were scanned into a photo CD (Eastman Kodak Co., Rochester, New York, USA) and quantification was performed by a blinded investigator on a Macintosh computer using the public domain National Institutes of Health Image program (version 1.60; NIH, Bethesda, Maryland, USA). Total area, in arbitrary pixel units, is reported for each mouse. Based on 
studies using standardized units of measurement superimposed onto the slides, 12,210 pixels $=1 \mathrm{~mm}^{2}$. Determination of levels of MMPs, RAGE, and EN-RAGEs in gingival samples. For detection of MMPs, gingival extracts were prepared as described previously (26). Briefly, gingival tissue pooled from sufficient numbers of mice per condition to normalize for final weight was homogenized in buffer containing Tris- $\mathrm{HCl}, \mathrm{NaCl}$, $\mathrm{CaCl}_{2}$, and urea, and rocked overnight at $4{ }^{\circ} \mathrm{C}$. After centrifugation, the supernatant was dialyzed versus 20,000 volumes of buffer containing Tris- $\mathrm{HCl}, \mathrm{NaCl}$, and $\mathrm{CaCl}_{2}$ over 3 days. Then $\left(\mathrm{NH}_{4}\right)_{2} \mathrm{SO}_{4}$ was added to the dialyzed material to achieve $60 \%$ saturation, and the mixture was incubated overnight at $4^{\circ} \mathrm{C}$. After centrifugation, the pellet was solubilized in buffer containing Tris- $\mathrm{HCl}, \mathrm{NaCl}, \mathrm{CaCl}_{2}$, and Brij 35 . The solubilized material was then dialyzed versus 20,000 volumes in the dialysis buffer over 2 days. Samples retrieved after dialysis were adjusted for total protein concentration (measured with the Bio-Rad protein assay; Bio-Rad Laboratories Inc., Hercules, California, USA) and subjected to electrophoresis on Tris-glycine gels (10\%; Novex, San Diego, California, USA). Contents of the gels were then transferred to nitrocellulose membranes (Novex), and immunoblotting was performed using mouse monoclonal anti-MMP 9 and anti-MMP 3 IgG (Oncogene Research Products, Cambridge, Massachusetts, USA). Rabbit anti-mouse IgG labeled with horseradish peroxidase (Sigma) was employed to identify the binding sites of primary antibody. In the case of RAGE or EN-RAGEs, gingival tissue, stored previously at $-80^{\circ} \mathrm{C}$, was thawed and homogenized in PBS containing a protease inhibitor mixture (Boehringer Mannheim Biochemicals Inc., Indianapolis, Indiana, USA). Protein concentration was measured as above, and samples were subjected to electrophoresis on Trisglycine gels (10\% and $18 \%$, respectively). Contents of the gels were then transferred to nitrocellulose membranes (Novex), and immunoblotting was performed with rabbit anti-murine RAGE IgG (22) or rabbit antibovine EN-RAGE (23) (the latter cross-reacts with the murine antigens), followed by goat anti-rabbit IgG labeled with horseradish peroxidase. In all cases, the enhanced chemiluminescence (ECL) detection system (Amersham-Pharmacia Biotech, Piscataway, New Jersey, USA) was used to indicate sites of primary/secondary antibody binding. Densitometry was performed using the ImageQuant software (Molecular Dynamics, Foster City, California, USA).

Determination of gelatinolytic activity in gingival samples. Gingival extracts, prepared as described above for detection of MMP protein, were subjected to electrophoresis on Tris-glycine gels (10\%) containing gelatin $(0.1 \%)$ according to the manufacturer's instructions (Novex). After staining in Coomassie brilliant blue $(0.5 \%)$, gels were dried, and densitometric analysis was performed using ImageQuant.

Detection of proinflammatory mediators in gingival tissue. Gingival tissue was prepared as above for detection of
RAGE and EN-RAGEs. The extracts were assayed for levels of TNF- $\alpha$ and IL- 6 by ELISA according to the manufacturer's instructions (R\&D Systems, Minneapolis, Minnesota, USA).

Analysis of gingival tissue for AGEs. At sacrifice, gingival tissue was removed, fixed in buffered formalin (10\%), and then paraffin-embedded sections, $5-\mu \mathrm{m}$ thick, were prepared. Immunohistochemistry was performed using affinity-purified rabbit anti-AGE IgG raised against carboxymethyl(lysine) (CML) modifications of proteins (29). CML-modifications of proteins, the predominant AGEs occurring in vivo, have been demonstrated to be specific AGE signal-transducing ligands of RAGE (29). In addition, for each condition sections were stained with nonimmune rabbit IgG at the same concentration as immune IgG. Detection of sites of primary antibody binding was accomplished using secondary antibodies and reagents in the ExtrAvidin Alkaline Phosphatase Staining Kit (Sigma). Quantification of immunohistochemistry was then performed as follows: multiple sections $(5-10)$ per condition were imaged under high power $(\times 200)$ using a Zeiss microscope and attached Sony video camera. Images were analyzed with respect to staining intensity on a Macintosh computer using the NIH Image program. Specifically, sections immunostained with nonimmune IgG for every condition were used to correct for background and nonspecific staining and to set the threshold above which staining of immune IgG sections was considered positive and specific for CML-AGE modifications. Results are presented as the percent above nondiabetic MSA-treated animals.

Statistical analysis. In all cases, mean \pm SE is reported. Statistical analysis was performed using Statview 4.01 (Abacus Concepts, Inc., Berkeley, California, USA) for Macintosh. When only 2 groups were compared, a 2tailed student's $t$ test was used. Multiple comparisons of bone loss measurements were performed by ANOVA. Dunnett's $t$ test was used for post hoc comparisons. $P$ values less then 0.05 were considered statistically significant.

\section{Results}

We demonstrated previously that sustained hyperglycemia in C57BL/6 mice inoculated with the periodontal pathogen $P$. gingivalis resulted in significantly increased alveolar bone loss 2 months after infection (26). To test the hypothesis that interaction of AGEs and/or EN-RAGEs with RAGE on critical cells in diabetic periodontium accounted for, at least in part, exaggerated inflammation and excessive alveolar bone loss, mice were rendered diabetic with streptozotocin and 1 month later were inoculated with $P$. gingivalis. Commencing immediately after inoculation, mice were treated once daily with intraperitoneal injections of either murine sRAGE or vehicle (MSA) for 2 months. At that time point, alveolar bone loss, defined as the area between the CEJ and BC, in diabetic MSA-treated mice was 6,272 \pm 81 pixels (mean \pm SE; Figure 1, a and c), significantly 
$\mathbf{a}$
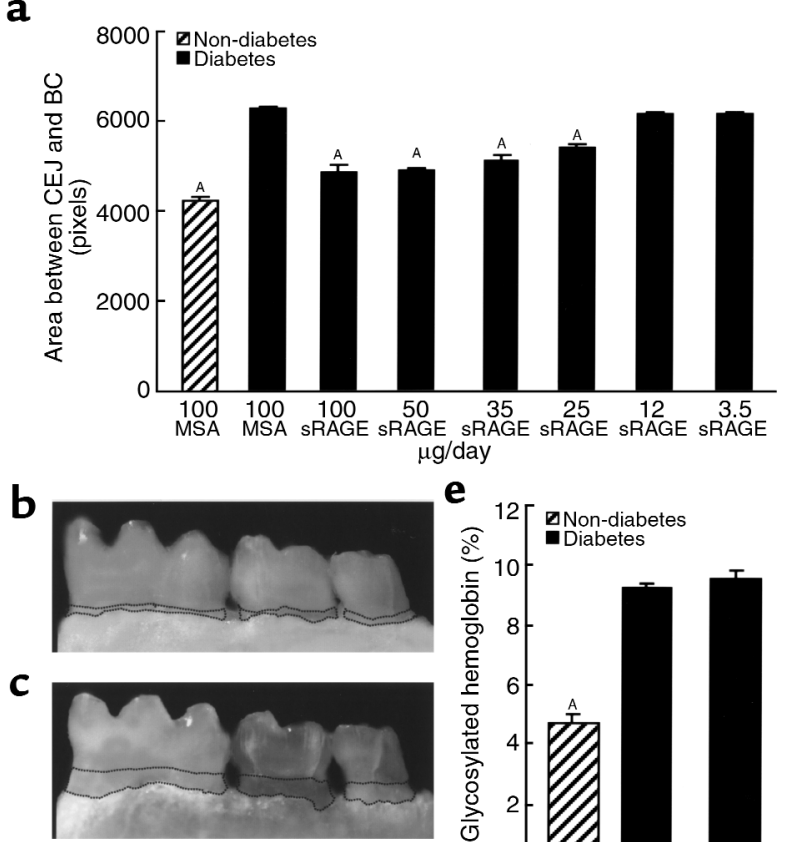

d

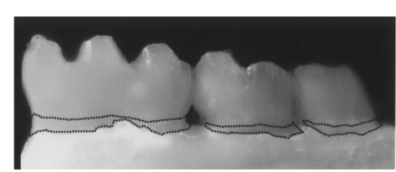

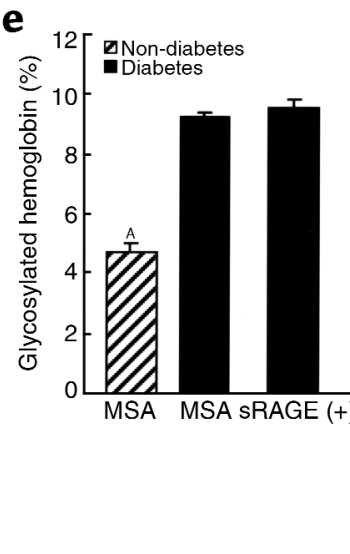

\section{Figure 1}

Administration of murine sRAGE results in dose-dependent suppression of alveolar bone loss in diabetic mice. (a) Alveolar bone loss. Male C57BL/6J mice were rendered diabetic with streptozotocin or treated with vehicle, citrate buffer alone. One month after documentation of diabetes or control state, all mice were inoculated with $P$. gingivalis, strain 381, as described in Methods. Immediately after inoculation, diabetic animals (filled bars) were treated with either MSA, $100 \mu \mathrm{g} /$ day $(n=11)$, or sRAGE, $100 \mu \mathrm{g} /$ day $(n=5)$, sRAGE, $50 \mu \mathrm{g} /$ day $(n=13)$, sRAGE, $35 \mu \mathrm{g} /$ day $(n=12)$, sRAGE, $25 \mu \mathrm{g} /$ day $(n=11)$, sRAGE, $12 \mu \mathrm{g} /$ day $(n=13)$, or sRAGE, $3.5 \mu \mathrm{g} /$ day $(n=12)$ for 2 months. Similarly, non-diabetic mice (hatched bar) were treated with MSA, $100 \mu \mathrm{g} /$ day $(n=12)$. Upon sacrifice, defleshed mandibles were assessed for extent of alveolar bone loss by morphometric analysis. Results are reported as mean arbitrary pixels per condition \pm SE. In these studies, 12,210 pixels $=1 \mathrm{~mm}^{2}$. Statistical analyses are as follows: diabetes/MSA vs. nondiabetes/MSA, $P=0.009$; diabetes/MSA vs.: sRAGE 25, 35, or 50 $\mu \mathrm{g} /$ day, $P<0.00001$; sRAGE $100 \mu \mathrm{g} /$ day, $P<0.0002$; sRAGE, 3.5 $\mu \mathrm{g} /$ day, $P=0.28$; and vs. sRAGE, $12 \mu \mathrm{g} /$ day, $P=0.20$. AStatistically significant differences compared with diabetes/MSA. (b-d). Representative photographs of mandibles. The lingual surfaces of posterior teeth are shown in defleshed half mandibles obtained from a non-diabetic mouse treated with MSA (b) and diabetic mice treated with MSA $(\mathbf{c})$ or $\operatorname{SRAGE}(+)(\mathbf{d})$. The outlined areas between the $C E J$ and $B C$ were computer analyzed and measured to evaluate differences in alveolar bone loss between groups. (e) Measurement of glycosylated hemoglobin. At the time of sacrifice, lysates of red blood cells were prepared as described and percent of glycosylated hemoglobin determined. The mean \pm SE is reported. Statistical analyses are as follows: non-diabetes/MSA vs. diabetes/MSA, $P=0.005$; diabetes/MSA vs. diabetes/sRAGE $(+), P=$ 0.5 ; and diabetes/sRAGE $(+)$ vs. non-diabetes/MSA, $P=0.005$. AStatistically significant differences compared with diabetes/MSA or diabetes/sRAGE $(+)$.

greater than that observed in non-diabetic mice $(4,234 \pm$ 114 pixels; Figure 1, a and b). Upon administration of sRAGE, diminished bone loss was observed. Alveolar bone loss in diabetic mice receiving sRAGE $25,35,50$, or $100 \mu \mathrm{g}$ per day was significantly suppressed $(5,437 \pm 74$, $5129 \pm 117,4,912 \pm 39$, and 4,879 \pm 151 pixels, respectively) when compared with diabetic mice receiving MSA; $P$ values were less than 0.0002 in all cases (Figure $1 \mathrm{a}, \mathrm{d}$, and $c$, respectively). The effects of sRAGE were dosedependent. At lower doses of sRAGE ( 3.5 and $12 \mu \mathrm{g}$ per day), levels of bone loss were similar to those observed in MSA-treated diabetic mice $(6,149 \pm 47$ and 6,147 \pm 79 pixels, respectively; Figure 1a). Because these latter 2 doses of sRAGE were not effective in preventing excessive alveolar bone loss, they are herein noted as sRAGE(-); those higher doses effective in reducing bone loss are noted as sRAGE $(+)$.

Importantly, the beneficial effects of sRAGE in suppressing alveolar bone loss were independent of the level of glycemia. Mean levels of glycosylated hemoglobin (note that these moieties are early glycation products, not AGEs) were not significantly different in sRAGE(+)-treated diabetic mice than levels observed in MSA-treated diabetic animals $(9.55 \pm 0.34 \%$ vs. $9.28 \pm$ $0.14 \%$, respectively; Figure 1e).

Since cytokines and MMPs are implicated in the soft tissue injury and bone loss that are pathognomonic of periodontal disease (9-10, 30-32), we assessed the levels of these mediators in gingival tissue. We first studied levels of MMP protein in gingival extracts. Compared with non-diabetic mice treated with MSA, diabetic mice demonstrated an approximately 7.3-fold increase in levels of MMP-9 as determined by immunoblotting (Figure 2a, lanes 1 and 2, respectively). In sRAGE(+)-treated diabetic animals, levels of MMP-9 were reduced 47\% (Figure 2a, lane 4). In contrast, in sRAGE(-)-treated mice, levels of MMP-9 were reduced only $19 \%$ (Figure 2a, lane 3). We similarly assessed levels of MMP-3 in gingival extracts. An approximately 3.9-fold increase in levels of MMP-3 by immunoblotting was observed in diabetic mice compared with non-diabetic controls, both receiving MSA (Figure $2 \mathrm{~b}$, lanes 2 and 1 , respectively). In the presence of effective doses of sRAGE, levels of MMP-3 were reduced $70 \%$ in diabetic gingival extracts (Figure $2 \mathrm{~b}$, lane 4 ) and $45 \%$ in diabetic mice treated with the lower doses of sRAGE (Figure 2b, lane 3).

Consistent with these observations, MMP-2 activity, as assessed in gingival extracts by zymography, was enhanced 3.4-fold in diabetic, MSA-treated mice compared with non-diabetic controls (Figure $2 \mathrm{c}$ and inset, lanes 2 and 1, respectively). MMP-2 activity was reduced $50 \%$ in mice treated with higher, effective doses of sRAGE, but only $17 \%$ in mice treated with lower doses of sRAGE (Figure $2 \mathrm{c}$ and inset, lanes 4 and 3, respectively).

TNF- $\alpha$ is an important proinflammatory cytokine associated with tissue destruction in periodontitis (30-32). Levels of TNF- $\alpha$ in gingival tissue extracts from diabetic MSA-treated mice $(n=9)$ were signifi- 

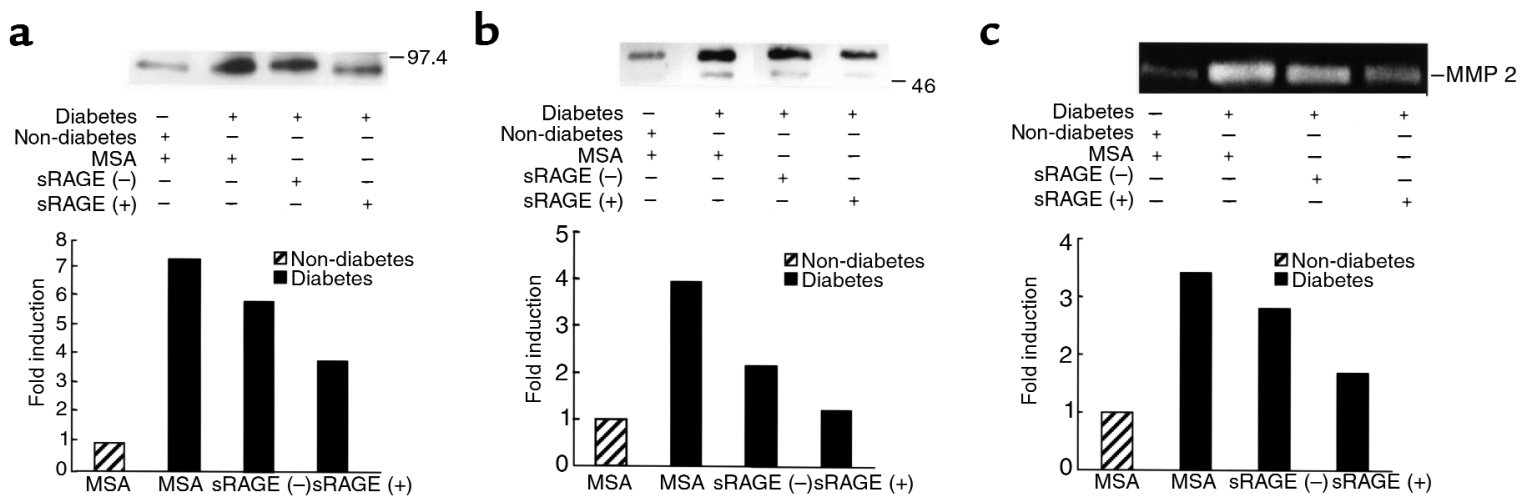

Figure 2

Administration of sRAGE to diabetic mice results in diminished levels of MMP protein and activity. (a, b) Immunoblotting. (a) MMP-9. Gingival tissue extracts were prepared as described and total protein $(150 \mathrm{ng})$ was subjected to electrophoresis on Tris-glycine gels. The contents of the gels were transferred to nitrocellulose membranes, and immunoblotting was performed using mouse monoclonal anti-MMP 9 (2 $\mu \mathrm{g} / \mathrm{mL}$ ). Densitometry was performed using ImageQuant. Molecular weight markers ( $\mathrm{kDa}$ ) are indicated at the right of the immunoblot. In these experiments, intensity of the band obtained from gingival extract of non-diabetic MSA-treated animals was arbitrarily defined as 1 . These experiments were performed 3 times with analogous results. (b) MMP-3. These experiments were performed as described in a; final concentration of anti-MMP-3 IgG was $1 \mu \mathrm{g} / \mathrm{mL}$. (c) Zymography. At sacrifice, gingival extracts were prepared as described. Two hundred twenty nanograms of total protein per sample was subjected to chromatography onto gels containing gelatin (0.1\%); bands representing MMP-2 are indicated. Densitometric analysis was performed and is demonstrated in the inset. Bands from non-diabetic mice treated with MSAwere arbitrarily defined as 1 . These experiments were performed 3 times with analogous results.

cantly increased compared with levels observed in nondiabetic MSA-treated controls $(n=10 ; 6.4 \pm 0.29$ vs. 4.7 $\pm 0.32 \mathrm{ng} / \mu \mathrm{g}$ tissue; $P=0.00003)$. In diabetic sRAGE $(+)$ treated mice $(n=8)$, levels of TNF- $\alpha$ were diminished $40 \%$ compared with MSA-treated diabetic animals ( 3.8 \pm 0.58 vs. $6.4 \pm 0.29 \mathrm{ng} / \mu \mathrm{g}$ tissue; $P=0.001)$. Indeed, levels of TNF- $\alpha$ in diabetic sRAGE(+)-treated mice were comparable to those in non-diabetic animals. In contrast, however, in sRAGE $(-)$-treated diabetic mice $(n=$ 11) levels of TNF- $\alpha$ were only suppressed $20 \%$ compared with MSA-treated diabetic mice (5.1 \pm 0.3 vs. 6.4 $\pm 0.29 \mathrm{ng} / \mu \mathrm{g}$ tissue; $P=0.07$ ).

We also assessed levels of IL-6 in gingival extracts, because it has been implicated in a number of proinflammatory events, including bone-resorptive activity $(33,34)$. Even though levels of IL-6 in gingival tissue, in general, demonstrated some variability, an approximately 1.7 -fold increase in the level of IL- 6 was noted in diabetic MSA-treated mice $(n=5)$ compared with non-diabetic controls $(n=7 ; 4.98 \pm 1.4$ vs. $3.00 \pm 0.7 \mathrm{ng} / \mu \mathrm{g}$ gingival tissue; $P=0.34)$. A trend toward decreased levels of IL- 6 was noted in $\operatorname{sRAGE}(+)$-treated diabetic mice $(n=6 ; 3.98 \pm 1.1$ $\mathrm{ng} / \mu \mathrm{g}$ tissue) compared with MSA-treated diabetic mice $(P=0.84)$. In contrast, levels of gingival IL-6 were not decreased in SRAGE $(-)$-treated mice $(n=6$; $5.53 \pm 1.7 \mathrm{ng} / \mu \mathrm{g}$ tissue) compared with MSA-treated diabetic animals $(P=0.48)$.

Since the promoter of RAGE is enriched in elements tightly linked to the inflammatory response, such as NF- KB (35), and since sRAGE prevents access of ligand to the cell-surface receptor, we hypothesized that decreased levels of RAGE itself might ensue upon administration of sRAGE. Consistent with these con- cepts, immunoblotting of gingival extracts revealed increased RAGE protein in diabetic compared with non-diabetic MSA-treated mice (Figure 3a); levels of tissue RAGE were suppressed, however, in mice treated with sRAGE (Figure 3a).

Both AGEs and/or EN-RAGEs are putative ligands promoting RAGE activation. We thus assessed their levels in gingival tissue. Compared with non-diabetic mice, levels of EN-RAGEs were increased $\approx 2$-fold in diabetic MSA-treated mice (Figure $3 \mathrm{~b}$ ). Significant suppression of EN-RAGE levels was observed in gingival tissue of mice treated with sRAGE(-) or sRAGE(+) (Figure $3 b)$. Furthermore, consistent with earlier observations (26), gingival tissue from diabetic MSA-treated mice demonstrated enhanced accumulation of AGEs compared with non-diabetic MSA-treated controls, especially within the vascular structures and surrounding epithelial and connective tissues (Figure 3, e and d, respectively, and Figure $3 \mathrm{~h}$ ). Accumulation of epithelial/connective tissue and vascular AGE was significantly suppressed in diabetic sRAGE $(+)$ mice; indeed these levels were not significantly different than those observed in non-diabetic MSA-treated mice (Figure $3, \mathrm{~g}$ and $\mathrm{d}$, respectively, and Figure $3 \mathrm{~h}$ ). In contrast, accumulation of gingival AGE retrieved from sRAGE(-)-treated diabetic mice was not different than that retrieved from diabetic MSA-treated mice (Figure 3 , f and e, respectively, and Figure $3 \mathrm{~h}$ ).

\section{Discussion}

Recent concepts have emerged linking the pathogenesis of certain diabetic complications to enhanced inflammatory responses. In such settings, sustained cellular activation often leads not to repair, but rather to 
Figure 3

Immunoblotting for RAGE (a) and EN-RAGEs (b) and immunohistochemistry for AGEs ( $\mathbf{c}-\mathbf{h})$. At sacrifice, gingival tissue was removed and was either prepared for immunoblotting as described above or fixed in buffered formalin (10\%). Paraffin-embedded sections, $5 \mu \mathrm{m}$ thick, were prepared for immunohistochemistry. Immunoblotting was performed with rabbit anti-murine RAGE IgG (4.5 $\mu \mathrm{g} / \mathrm{mL})$ or rabbit anti-EN-RAGE IgG $(2 \mu \mathrm{g} / \mathrm{mL})$ as above. Molecular weight markers $(\mathrm{kDa})$ are indicated on the right side of the immunoblot. Immunohistochemistry is shown for a representative section from a diabetic MSA-treated mouse using nonimmune rabbit $\operatorname{lgG}(1 \mu \mathrm{g} / \mathrm{mL}$ in c) or affinity-purified anti-AGE IgG $(1 \mu \mathrm{g} / \mathrm{mL}$ in $\mathbf{d}-\mathbf{g})$. Samples are as follows: non-diabetes/MSA, d; diabetes/MSA, e; diabetes/sRAGE(-), f; and diabetes/sRAGE(+), g. Scale bar: $45 \mu \mathrm{m}$. In $\mathbf{h}$, the results of quantitative analysis of the immunohistochemistry, performed as described above, are shown.

tissue injury and chronic disease (36). For example, elevated levels of plasma $\mathrm{C}$-reactive protein were found in patients with type 1 diabetes, along with increased levels of circulating von Willebrand factor and soluble VCAM-1 (37). Together, these factors were associated with increased urinary albumin excretion, a marker of diffuse vascular dys-

function associated with long-term cardiovascular complications $(38,39)$. In this context, a plethora of studies have suggested a direct link between enhanced levels of inflammatory mediators and tissue-destructive enzymes and severe periodontal disease in diabetes. Our present findings demonstrate that blockade of RAGE results in suppression of both alveolar bone loss and markers of cellular activation/tissue-destructive properties in diabetic mice infected with $P$. gingivalis. These data further support the concept that factors central to diabetes, such as activation of RAGE, account for exaggerated inflammation and tissue destruction in diabetic periodontium and thus provide novel insights into the molecular mechanisms underlying the pathogenesis of diabetes-associated periodontal disease.

The implications of diabetes-associated periodontitis extend to the general health of the host, because recent findings have linked periodontal disease to increased incidence of disorders, such as atherosclerosis and cerebrovascular events, in which inflammation plays an important role (40-44). Because diabetes itself is a risk factor for aggressive vascular disease $(45,46)$, these considerations highlight a sustained cascade of cellular activation, linking diabetes, periodontal and systemic inflammation, and atherosclerosis. Indeed, studies suggesting that treatment of periodontal disease improved glycemic control in diabetic individuals, as measured by reduction of glycated hemoglobin, close this loop and underscore an inextricable link b

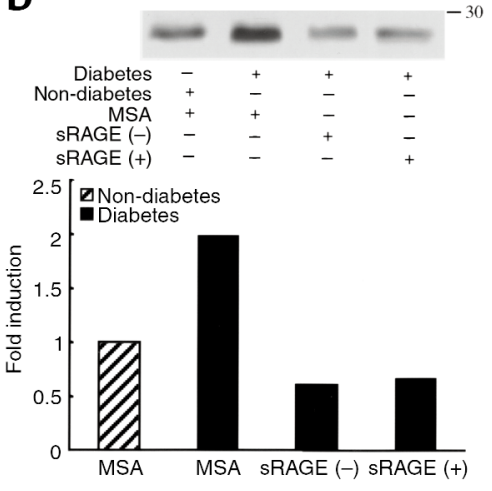

d

e

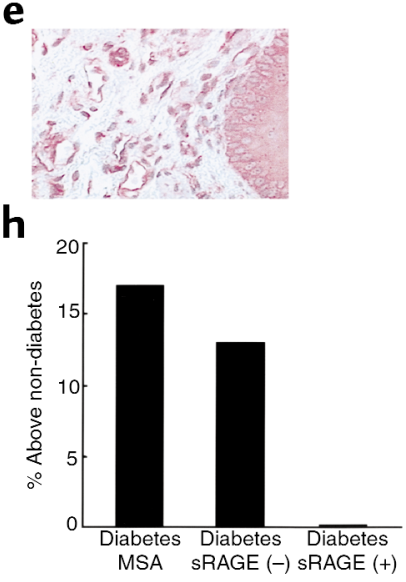

between diabetes and the inflammatory response (47). Furthermore, the present findings identify an important role for RAGE in these processes, because this and previous studies have indicated that blockade of RAGE suppresses inflammatory complications of diabetes, such as accelerated atherosclerosis (22) and, now, periodontitis-associated alveolar bone loss.

Importantly, the present studies provide new insights into the biology of RAGE and its ligands in distinct settings. Our data suggest that AGEs are a critical ligand for RAGE in diabetic periodontium because decreasing levels of gingival AGEs paralleled the observed suppression of alveolar bone loss with sRAGE treatment. In contrast, levels of gingival EN-RAGEs were similarly suppressed in both diabetic sRAGE(-) and sRAGE(+) mice. Of course, we cannot definitively rule out the possibility that differences in cellular localization of EN-RAGEs (that is, within the intracellular or extracellular space) may have existed between the 2 groups of mice. Nevertheless, the data suggest that chronic accumulation of AGEs, signal-transducing ligands of RAGE, provided a potent and sustained stimulus to activation of the receptor and of tissuedestructive inflammatory cascades in diabetic periodontium. In addition, strongly supportive of the central role for AGEs in diabetes-associated periodontitis is our finding that administration of sRAGE, $35 \mu \mathrm{g} / \mathrm{day}$, to euglycemic mice infected with $P$. gingivalis did not result in suppressed alveolar bone loss in this model $(4,056 \pm 111$ vs. $4,234 \pm 114$ pixels, respectively). 
Our present findings possess clinical relevance as well, because current strategies to minimize destruction of supportive tissues in diabetic periodontitis lack direct targeting of the host response. In fact, current therapeutic modalities largely target the microbial component of periodontal disease (48). In this context, efforts are aimed at reducing microbial plaque from the subgingival environment by nonsurgical or surgical debridement of the root surface. However, especially for patients with poor glycemic control, extensive surgery may prove not to be an optimal course of treatment, because impaired wound healing is certainly an established feature of diabetes.

Previous studies have identified non-antimicrobial mechanisms by which agents such as nonsteroidal antiinflammatory drugs, low-dose doxycycline and chemically modified tetracyclines (CMTs), antagonists of proinflammatory cytokines, and inhibitors of cyclooxygenase- 2 may exert beneficial effects in periodontal disease (49-52). Specifically, doxycycline and CMTs have demonstrated promise in enhancing collagen production and suppressing MMP synthesis/activation in cellbased systems and diabetic organisms (28, 53, 54). Indeed, recent observations that antagonists of IL-1 and TNF diminish the progression of inflammatory cell infiltration toward alveolar bone in a primate model of experimental periodontitis strongly support the notion that suppression of inflammatory mediators is critical in interrupting the tissue-destructive course of periodontal disease (55).

Our observations highlight a central pathogenic role for RAGE in the development of accelerated periodontal inflammation and alveolar bone loss in diabetic rodents. Although our studies demonstrated reduced alveolar bone loss in the presence of sRAGE, it is likely that administration by the intraperitoneal route is not the most optimal. These considerations underscore the possibility that future efforts to block periodontal RAGE might best be performed by local delivery at the site of the periodontal lesion, a feat not technically feasible in the murine model because of the small size of the involved tissues.

Taken together, the present findings delineate a critical link between oral infection, exaggerated inflammatory host responses, and destruction of alveolar bone in diabetes. These observations highlight a central pathogenic role for RAGE in these settings and identify RAGE as a directed, logical target for suppression of periodontal destruction in diabetes. Indeed, optimal suppression of periodontal inflammation, in turn, is likely to preserve a range of homeostatic properties in the diabetic host.

\section{Acknowledgments}

The authors gratefully acknowledge the advice and suggestions of Lorne Golub and Maria Ryan. This work was supported, in part, by the Surgical Research Fund of the College of Physicians and Surgeons, Columbia University, and by grants from the US Public Health Service (DE11561 and HL60901), American Dental Association
Health Foundation, Juvenile Diabetes Foundation International, and the Council for Tobacco Research. A.M. Schmidt is a recipient of a Burroughs Wellcome Fund Clinical Scientist Award in Translational Research.

1. Cianciola, L., Park, B., Bruck, E., Mosorich, L., and Genco, R.J. 1982. Prevalence of periodontal disease in insulin-dependent diabetes mellitus. J. Am. Dent. Assoc. 104: 653-660.

2. Shlossman, M., Knowler, W.C., Pettitt, D.J., and Genco, R.J. 1990. Type 2 diabetes mellitus and periodontal disease. J. Am. Dent. Assoc. 121:531-536

3. Löe, H. 1993. Periodontal disease. The sixth complication of diabetes mellitus. Diabetes Care. 16:329-334.

4. Manouchehr-Pour, M., Spagnuolo, P.J., Rodman, H.M., and Bissada, N.F. 1981. Comparison of neutrophil chemotactic response in diabetic patients with mild and severe periodontal disease. J. Periodontol. 52:410-414.

5. McMullen, J.A., Van Dyke, T.E., Horoszewicz, H.U., and Genco, R.J. 1981. Neutrophil chemotaxis in individuals with advanced periodontal disease and a genetic predisposition to diabetes mellitus. J. Periodontol. 52:167-173.

6. Ramamurthy, N.S., and Golub, L.M. 1983. Diabetes increases collagenase activity in extracts of rat gingiva and skin. J. Periodontal Res. 18:23-30.

7. Sasaki, T., Ramamurthy, N.S., Yu, Z., and Golub, L.M. 1992. Tetracycline administration increases protein (presumably procollagen) synthesis and secretion in periodontal ligament fibroblasts of streptozotocininduced diabetic rats. J. Periodontal Res. 27:631-639.

8. Molvig, J., et al. 1988. Endotoxin-stimulated human monocyte secretion of interleukin 1, tumor necrosis factor alpha, and prostaglandin $\mathrm{E}_{2}$ shows stable interindividual differences. Scand. J. Immunol. 27:705-716.

9. Salvi, G.E., et al. 1997. Monocytic TNF- $\alpha$ secretion patterns in IDDM patients with periodontal diseases. J. Clin. Periodontol. 24:8-16.

10. Salvi, G.E., et al. 1997. Inflammatory mediator response as a potential risk marker for periodontal diseases in insulin dependent diabetes mellitus patients. J. Periodontal Res. 68:127-135.

11. Brownlee, M., Cerami, A., and Vlassara, H. 1988. Advanced glycosylation end products in tissue and the biochemical basis of diabetic complications. N. Engl. J. Med. 318: 1315-1320.

12. Bucala, R., et al. 1994. Modification of low density lipoprotein by AGEs contributes to the dyslipidemia of diabetes and renal insufficiency. Proc. Natl. Acad. Sci. USA. 27:9441-9445.

13. Ruderman, N., Williamson, J., and Brownlee, M. 1992. Glucose and diabetic vascular disease. FASEB J. 6:2905-2914.

14. Schmidt, A.M., et al. 1992. Isolation and characterization of binding proteins for advanced glycosylation end products from lung tissue which are present on the endothelial cell surface. J. Biol. Chem. 267:14987-14997.

15. Neeper, M., et al. 1992. Cloning and expression of RAGE: a cell surface receptor for advanced glycosylation end products of proteins. J. Biol. Chem. 267:14998-15004.

16. Brett, J., et al. 1993. Tissue distribution of the receptor for advanced glycation end products (RAGE): expression in smooth muscle, cardiac myocytes, and neural tissue in addition to the vasculature. Am. J. Pathol. 143:1699-1712.

17. Schmidt, A.M., Yan, S.D., and Stern, D. 1995. The dark side of glucose (News and Views). Nat. Med. 1:1002-1004.

18. Owen, W.F., Jr., et al. 1998. $\beta_{2}$-Microglobulin modified with advanced glycation end products modulates collagen synthesis by human fibroblasts. Kidney Int. 53:1365-1373.

19. Schmidt, A.M., Yan, S.D., Wautier, J.L., and Stern, D. 1999. Activation of receptor for advanced glycation end products: a mechanism for chronic vascular dysfunction in diabetic vasculopathy and atherosclerosis. Circ. Res. 84:489-497.

20. Seppala, B., Sorsa, T., and Ainamo, J. 1997. Morphometric analysis of cellular and vascular changes in gingival connective tissue in long-term insulin-dependent diabetes. J. Periodontol. 68:1237-1245.

21. Wautier, J.L., et al. 1996. Receptor-mediated endothelial cell dysfunction in diabetic vasculopathy: soluble receptor for advanced glycation end products blocks hyperpermeability. J. Clin. Invest. 97:238-243.

22. Park, L., et al. 1998. Suppression of accelerated diabetic atherosclerosis by soluble receptor for AGE (sRAGE). Nat. Med. 4:1025-1031.

23. Hofmann, M.A., et al. 1999. RAGE mediates a novel proinflammatory axis: a central cell surface receptor for S100/calgranulin polypeptides. Cell. 97:889-901.

24. Schafer, B.W., and Heinzmann, C.W. 1996. The S100 family of EF-hand calcium-binding proteins: functions and pathology. Trends Biochem. Sci. 21:134-140.

25. Zimmer, D.B., Cornwall, E.H., Landar, A., and Song, W. 1995. The S100 protein family: history, function, and expression. Brain Res. Bull. 37:417-429. 
26. Lalla, E., Lamster, I.B., Feit, M., Huang, L., and Schmidt, A.M. 1998. A murine model of accelerated periodontal disease in diabetes. J. Periodontal Res. 33:387-399.

27. Evans, R.T., et al. 1992. Periodontopathic potential of two strains of Porphyromonas gingivalis in gnotobiotic rats. Arch. Oral. Biol. 37:813-819.

28. Chang, K.M., et al. 1994. Tetracyclines inhibit Porphyromonas gingivalisinduced alveolar bone loss in rats by a non-antimicrobial mechanism. $J$. Periodontal Res. 29:242-249.

29. Kislinger, T., et al. 1999. N(epsilon)-(carboxymethyl)lysine adducts of proteins are ligands for receptor for advanced glycation end products that activate cell signaling pathways and modulate gene expression. J. Biol. Chem. 274:31740-31749.

30. Lamster, I.B., and Novak, M.J. 1992. Host mediators in gingival crevicular fluid: implications for the pathogenesis of periodontal disease. Crit. Rev. Oral Biol. Med. 3:31-60.

31. Stashenko, P., Jandinski, J.J., Fujiyoshi, P., Rynar, J., and Socransky, S.S. 1991. Tissue levels of bone resorptive cytokines in periodontal disease. J. Periodontol. 62:504-509.

32. Reynolds, J.J., and Meikle, M.C. 1997. Mechanisms of connective tissue matrix destruction in periodontitis. Periodontol. 2000. 14:144-157.

33. Adebanjo, O.A., et al. 1998. Mode of action of IL- 6 on mature osteoclasts. Novel interactions with extracellular calcium-sensing in the regulation of osteoclastic bone resorption. J. Cell Biol. 142:1347-1356.

34. Manolagas, S.C. 1998. The role of IL- 6 type cytokines and their receptors in bone. Ann. NY Acad. Sci. 840:194-204.

35. Li, J., and Schmidt, A.M. 1997. Characterization and functional analysis of the promoter of RAGE, the receptor for advanced glycation endproducts. J. Biol. Chem. 272:16498-16506.

36. Pickup, J.C., and Crook, M.A. 1998. Is type 2 diabetes mellitus a disease of the innate immune system? Diabetologia. 41:1241-1248.

37. Schalkwijk, C.G., et al. 1999. Plasma concentration of C-reactive protein is increased in type 1 diabetic patients without clinical macroangiopathy and correlates with markers of endothelial dysfunction: evidence for chronic inflammation. Diabetologia. 42:351-357.

38. Jensen, T., Borch-Johnson, K., Kofoed-Enevoldsen, A., and Decker, T. 1987. Coronary heart disease in young type 1 diabetic patients with and without diabetic nephropathy: incidence and risk factors. Diabetologia. 30:144-148.

39. Messent, J., et al. 1992. Prognostic significance of microalbuminuria in insulin-dependent diabetes mellitus: a twenty-three year follow-up study. Kidney Int. 41:836-839.

40. Beck, J.D., Offenbacher, S., Williams, R., Gibbs, P., and Garcia, R. 1998 Periodontitis: a risk factor for coronary heart disease? Ann. Periodontol. 3:127-141

41. Meyer, D.H., and Fives-Taylor, P.M. 1998. Oral pathogens: from dental plaque to cardiac disease. Curr. Opin. Microbiol. 1:88-95.

42. Morrison, H.I., Ellison, L.F., and Taylor, G.W. 1999. Periodontal disease and risk of fatal coronary heart and cerebrovascular diseases. J. Cardiovasc. Risk. 6:7-11.

43. Destefano, F., Anda, R.F., Kahn, H.S., Williamson, D.F., and Russell, C.M. 1993. Dental disease and risk of coronary heart disease and mortality. BMJ. 306:688-691.

44. Ross, R. 1999. Atherosclerosis: an inflammatory disease. N. Engl. J. Med. 340:115-126.

45. Uusitupa, M.I., Niskanen, L.K., Siitonen, O., Voutilainen, E., and Pyorala, K. 1990. Five-year incidence of atherosclerotic vascular disease in relation to general risk factors, insulin level, and abnormalities in lipoprotein composition in non-insulin-dependent diabetic and non-diabetic subjects. Circulation. 82:27-36.

46. Manson, J.E., et al. 1991. A prospective study of maturity-onset diabetes mellitus and risk of coronary heart disease and stroke in women. Arch. Intern. Med. 151:1141-1147.

47. Grossi, S.G., et al. 1997. Treatment of periodontal disease in diabetics reduces glycated hemoglobin. J. Periodontol. 68:713-719.

48. World Workshop in Periodontics. 1996. Consensus report non-surgical pocket therapy: mechanical, pharmacotherapeutics, and dental occlusion. Ann. Periodontol. 2:581-588.

49. Williams, R.C., et al. 1989. Altering the progression of human alveolar bone loss with the non-steroidal anti-inflammatory drug flurbiprofen. J. Periodontol. 9:485-490.

50. Kornman, K.S., Blodgett, R.F., Brunsvold, M., and Holt, S.C. 1990. Effects of topical applications of meclofenamic acid and ibuprofen on bone loss, subgingival microbiota and gingival PMN response in the primate Macaca fascicularis. J. Periodontal Res. 25:300-307.

51. Golub, L.M., et al. 1998. Tetracyclines inhibit connective tissue breakdown by multiple non-antimicrobial mechanisms. Adv. Dent. Res. 12:12-26.

52. Noguchi, K., et al. 1996. Prostaglandin production via induction of cyclooxygenase- 2 by human gingival fibroblasts stimulated with lipopolysacharides. Inflammation. 5:555-568.

53. Craig, R.G., et al. 1998. A chemically modified tetracycline inhibits streptozotocin-induced diabetic depression of skin collagen synthesis and steady-state type I procollagen mRNA. Biochim. Biophys. Acta. 1402:250-260.

54. Golub, L.M., et al. 1983. Minocycline reduces gingival collagenolytic activity during diabetes. J. Periodontal Res. 18:516-526.

55. Graves, D.T., et al. 1998. Interleukin-1 and tumor necrosis factor antagonists inhibit the progression of inflammatory cell infiltration toward alveolar bone in experimental periodontitis. J. Periodontol. 69:1419-1425. 